\title{
The hunger games: sensing host arginine is essential for Leishmania
}

\author{
parasite virulence
}

Adele Goldman-Pinkovich ${ }^{11}$, Sriram Kannan ${ }^{2}$, Roni Nitzan-Koren ${ }^{1}$, Madhu Puri ${ }^{3}$, Yael BarAvraham $^{1}$, Jacqueline A. McDonald ${ }^{4}$, Aakash Sur ${ }^{4,5}$, Wen-Wei Zhang ${ }^{6}$, Greg Matlashewski ${ }^{6}$, Rentala Madhubala ${ }^{3}$, Shulamit Michaeli ${ }^{2}$, Peter J. Myler ${ }^{4,5,7}$ and Dan Zilberstein ${ }^{1 *}$

${ }^{1}$ Faculty of Biology, Technion - Israel Institute of Technology, Haifa, Israel

${ }^{2}$ The Mina and Everard Goodman Faculty of Life Sciences and Advanced Materials and Nanotechnology Institute, Bar-Ilan University, Ramat-Gan Israel

${ }^{3}$ School of Life Sciences, Jawaharlal Nehru University, New Delhi, India

${ }^{4}$ Center for Global Infectious Disease Research, Seattle Children's Research Institute, USA

${ }^{5}$ Department of Biomedical Informatics and Medical Education, University of Washington, Seattle, USA

${ }^{6}$ Department of Microbiology and Immunology, McGill University, Montreal, Canada

${ }^{7}$ Department of Global Health, University of Washington, Seattle, USA

${ }^{\mathrm{I}}$ Current address: Department of Immunology and infectious Diseases, Harvard T.H. Chan School of Public Health, Boston, USA

*Corresponding address: $\underline{\text { danz@technion.ac.il }}$ 


\section{Abstract}

Arginine homeostasis in lysosomes is critical for growth and metabolism of mammalian cells. They employ a specific sensor (SLC38A9) that monitors intra-lysosome arginine sufficiency and subsequently up-regulates cellular mTORC1 activity. Lysosomes of macrophages (phagolysosomes) are the niche where the parasitic protozoan Leishmania resides and causes important human disease. Several years ago, we discovered that upon arginine starvation, cultured Leishmania parasites promptly activate a MAPK2-mediated Arginine Deprivation Response (ADR) pathway, resulting in up-regulation of the Leishmania arginine transporter (AAP3), as well as a small group of other transporters. Significantly, ADR is also activated during macrophage infection, implying that the intracellular parasite actively depletes arginine within the host phagolysosome, likely to prevent mTORC1 activation and enhance intracellular development. We hypothesize that ADR-mediated upregulation of AAP3 activity is necessary to withstand the resultant arginine starvation. Both copies of the AAP3 genes are located (in tandem) on a tetrasomic chromosome (chr31), but only one (AAP3.2) is responsive to arginine deprivation. CRISPR/Cas9-mediated disruption of the AAP3 locus yielded mutants that retain a basal level of arginine transport (mediated by $A A P 3.1)$, but lack a functional copy of $A A P 3.2$ and are therefore not responsive to arginine starvation. While these mutants grow normally in culture as promastigotes, they were impaired in their ability to develop inside THP1 macrophages grown under physiological concentrations of arginine $(0.1 \mathrm{mM})$. However, flooding the macrophage growth medium with arginine $(1.5 \mathrm{mM})$ restored parasite infectivity and intracellular growth to that of wild type. The results indicate that inside the host macrophage, Leishmania must overcome the arginine "Hunger Games" by up-regulating transport of arginine via the ADR. Furthermore, the AAP3.2 mutants were $70-80 \%$ less virulent in Balb/C mice, showing, for the first time, 
bioRxiv preprint doi: https://doi.org/10.1101/751610; this version posted August 30, 2019. The copyright holder for this preprint (which was not certified by peer review) is the author/funder. All rights reserved. No reuse allowed without permission.

that the ability to monitor and respond to changes in host metabolite levels is essential for pathogenesis. 


\section{Introduction}

The lysosomes of phagocytic cells are where pathogens fight for life in a hostile environment, dependent on the host for essential nutrient supply. In the case of the obligatory intracellular parasite Leishmania, arginine availability is of particular interest because it is important for both host defense and parasite proliferation. Sensing of arginine levels in the lysosome lumen is a key mechanism that regulates mTORC1 activity in mammalian cells (Saxton and Sabatini, 2017). In macrophages, mTORC1 activation induces a Th1 response, resulting in production of cytotoxic nitric oxide from arginine (Weinstein et al., 2000). This pathway is the major means by which macrophages kill invading pathogenic microorganisms. Interestingly, Leishmania parasites are able to counteract this outcome by activating a Th2 response, directing arginine towards polyamine biosynthesis instead of NO production, thereby enabling the parasites to persist and cause long-term non-healing infections (Shahi et al., 2013)(Rath et al., 2014). However, both Th1 and Th2 responses result in arginine depletion in the macrophage phagolysosome, presenting an existential threat to their survival. In this study, we explore the mechanism that enables intracellular Leishmania to win this "Hunger Game".

Recently, our laboratory discovered that an MAPK2-mediated Arginine Deprivation Response (ADR) pathway is triggered immediately upon arginine starvation of the cultured extracellular insect-form (promastigote) Leishmania, resulting in up-regulation of arginine transporter (AAP3) mRNA and protein levels, with a concomitant increase in arginine transport efficiency (Goldman-Pinkovich et al., 2016). Significantly, the ADR is also activated during Leishmania infection of macrophages (Goldman-Pinkovich et al., 2016)'(Pawar et al., 2019), suggesting a role for intra-lysosomal arginine sensing in intracellular (amastigote) parasite survival and pathogenesis. In this study, we sought to 
bioRxiv preprint doi: https://doi.org/10.1101/751610; this version posted August 30, 2019. The copyright holder for this preprint (which was not certified by peer review) is the author/funder. All rights reserved. No reuse allowed without permission.

critically assess this hypothesis by creating mutants that are unable to up-regulate AAP3 expression after arginine starvation. 


\section{Results and discussion}

Two genes (AAP3.1 and AAP3.2) that are highly conserved (only three amino acid differences) within their coding sequence (CDS) and 5' untranslated region (UTR) but have very different 3' UTRs encode AAP3. However, only AAP3.2 is responsive to arginine deprivation (Darlyuk et al., 2009). Chromosome 31, where the two AAP3 genes occur in a tandem array, is tetraploid in L. donovani (Downing et al., 2011); making classical homologous gene replacement approaches cumbersome and tedious, especially since the plasticity of Leishmania genome enables parasites to retain an additional wild-type chromosome as well as those containing the selectable marker(s)(Laffitte et al., 2016). Therefore, we used the Leishmania-adapted CRISPR/Cas9 system(Zhang and Matlashewski, 2015) to expedite disruption of the AAP3 genes.

Wild type (WT) L. donovani was transfected (separately) with plasmids containing three 21-nt gRNAs (G1, G2 and G3) targeting the 5' end (+19, 85 and 173) of both AAP3.1 and AAP3.2 CDSs (Fig. 1A, top panel) and the cultures examined 6 weeks later for AAP3 protein levels in the presence or absence of arginine. While the G1-transfected cultures showed enhanced ADR-mediated increase in AAP3 levels compare to WT, the G2 and G3 cultures showed a diminished capacity to up-regulate AAP3 levels after arginine starvation (Fig. 1B, top left panel). The G3 culture was seeded onto agar plates and individual colonies

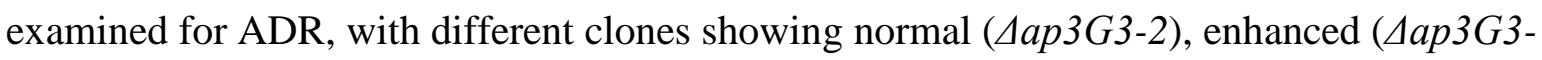
17), or reduced ( $\triangle a p 3 G 3-1)$ increases in AAP3 protein abundance compared to WT (Fig. 1B, top right panel). In order to increase the efficiency of CRISPR/Cas9-mediated gene disruption, $\triangle a p 3 G 3-1$ was transfected (four times at 3-day intervals) with a 61-nt "donor" oligonucleotide consisting of 25-nt flanking an 11-nt insertion with two in-frame stop codons at the Cas 9 cleavage site (Fig. 1A, bottom panel). Single colonies were isolated 3 weeks after transfection and examined for AAP3 expression levels after arginine deprivation. Two clones 
( $\triangle a p 3 D 6$ and $\triangle a p 3 D 10$ ) showed no increase in AAP3 protein abundance after arginine deprivation, while two others ( $\triangle a p 3 D 4$ and $D 7)$ showed a small (compared to WT) increase in AAP3 protein levels (Fig. 1B, bottom panel). Analyses of the initial rates of arginine transport confirmed that these mutants had lost all or some of their response to arginine deprivation (Figs. 1C and S1). While WT cells showed a 2.7-fold increase in the initial rate of arginine transport 2 hours after arginine starvation, most of the mutants showed little or no increase in transport rate after arginine starvation (1.5-, 1.2, 1.5- and 1.1-fold for $\triangle a p 3 D 4$, $\triangle a p 3 D 6, \triangle a p 3 D 7$ and $\triangle a p 3 D 10$, respectively). Interestingly, $\triangle a p 3 G 3-17$ showed a higher than WT (3.2-fold) increase in transport after arginine deprivation, consistent with its having additional copies of the AAP3.2 gene (see below).

Figure 1
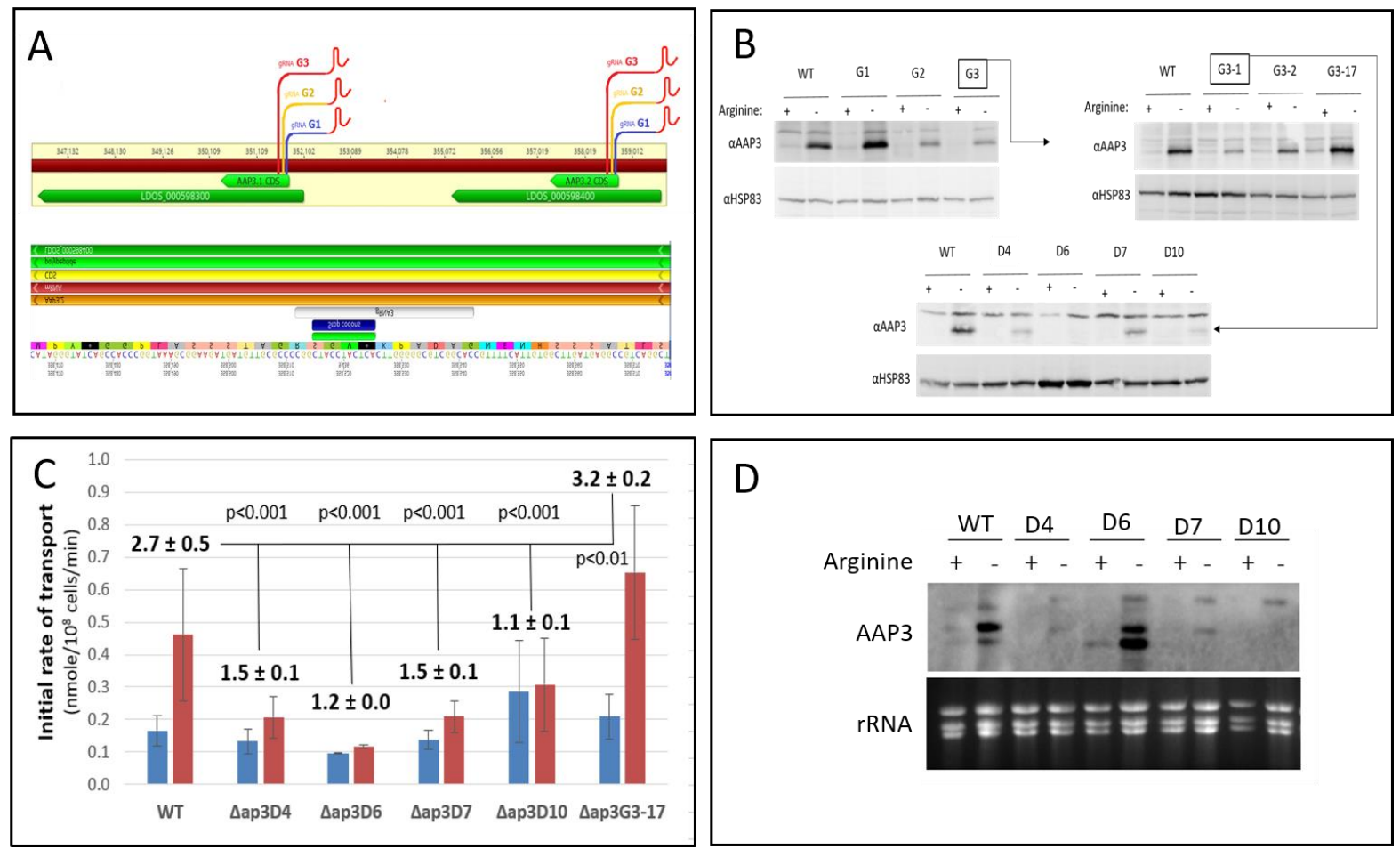
Whole genome sequencing was carried out to map the precise location of the CRISPR/Cas9-induced mutations in each cell-line. Illumina libraries were constructed and sequenced on a HiSeq 4000 using paired-end 75-bp reads with TruSeq standard primers.

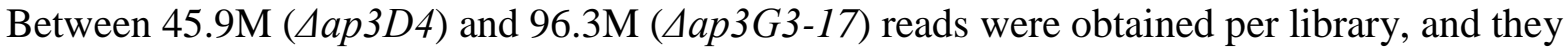
were aligned against an L. donovani $1 \mathrm{~S}$ genome sequence assembled from a combination of PacBio and Illumina reads (manuscript in preparation). As shown in Fig. 2A, changes in coverage (read counts) were seen in the $A A P 3$ locus of all mutants ( $\triangle a p 3 D 7$ is not shown since it was essentially identical $\triangle a p 3 D 4)$. As expected, the WT parent had four copies of both AAP3.1 and AAP3.2, as well as the genes flanking this locus (Fig. 2B). However, Lap3G3-1 and most of its progeny (D4 and D10) had substantially lower coverage in the sequence between the 5' UTR of AAP3.1 and the 3' end of AAP3.2, consistent with recombination near the double-strand break(s) introduced by Cas9 at the site of the gRNA-G3 sequence to create a AAP3.1/AAP3.2 fusion that contains the 5' UTR of AAP3.2 and the 3' UTR of AAP32, along with deletion of 3' UTR of AAP3.2and the ncRNA located in the intergenic region between the two AAP3 genes. Quantitation of the read counts (Fig. 2B) indicated that $\triangle a p 3 G 3-1$ retained two intact copies of $A A P 3.1$ and $A A P 3.2$, along with two copies of the AAP3.1/AAP3.2 fusion; Aap3G3-1/D4 (and $\triangle a p 3 G 3-1 / D 7$ ) retained one copy of the intact AAP3 locus (containing both AAP3.1 and AAP3.2) and three copies with the AAP3.1/AAP3.2 fusion; while in $\triangle a p 3 D 10$ all four copies of the AAP3 locus contain the AAP3.1/AAP3.2 fusion. However, the presence of a small number (102) of reads from this region in $\triangle a p 3 D 10$ suggests that some cells in this population may retain an intact copy of chr31 (although this could be due to presence of an extrachromosomal circle formed between $A A P 3.1$ and $A A P 3.2$ or contamination with another cell-line). In contrast, $\triangle a p 3 G 3-17$ showed higher coverage in this region, consistent with recombination between the two copies of the $A A P 3$ gene resulting in additional (2-3 for each copy of chr31) copies of AAP3.2 and the 
intergenic sequence (including the ncRNA). Interestingly, $\triangle a p 3 D 6$ shows slightly higher than WT levels of read coverage for the AAP3.1 gene, suggesting that it retained only one copy of chr31 with the AAP3.1/AAP3.2 fusion, along with three copies containing both $A A P 3.1$ and $A A P 3.2$ genes (one may contain an additional AAP3.2 gene). Eexamination of the read coverage in the gRNA-G3 region indicated that $~ 78 \%$ (418/536) contain an 11-bp insertion (with two in-frame stop codons), consistent with the hypothesis that $\triangle a p 3 D 6$ contained 7 copies of the AAP3 gene with stop codons that render them non-functional at the protein level and only one (or two) functional version(s). The inability of this mutant to present with even partial ADR on protein level such as that of mutants D4/D7 suggests that the functional copies are the fused AAP3.1/AAP3.2 which lack stop codons but also lack ADR capacity.

Figure 2

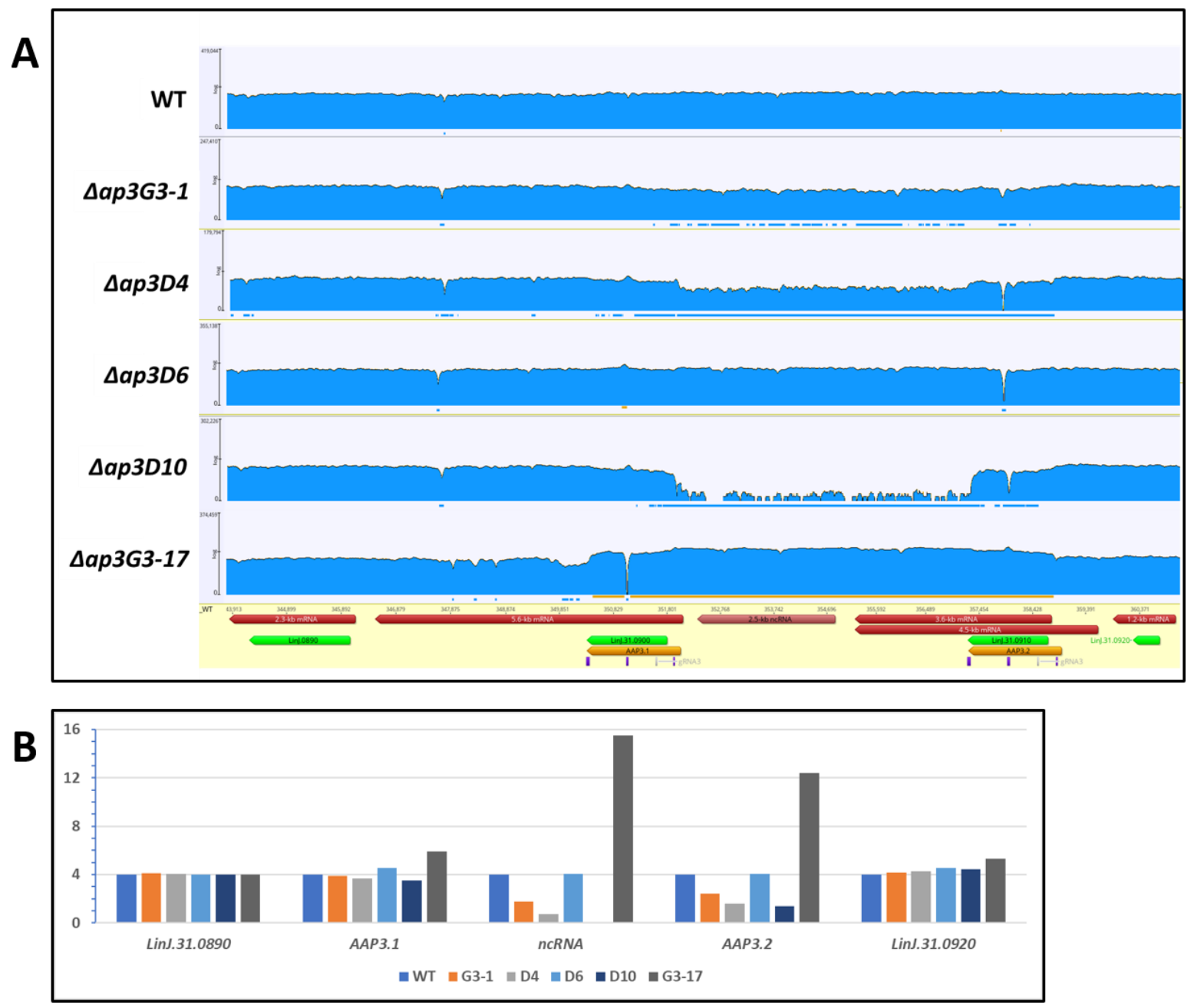


Northern analyses (Fig. 1D) confirmed the reduction in AAP3.2 gene copy number by showing that its mRNA levels after arginine deprivation were significantly lower than WT in the in $D 4, D 7$ and $D 10$ cell-lines. These changes paralleled the changes in protein abundance observed in Fig. 1B, confirming that AAP3.2 accounts for most (if not all) of the increase in arginine transport after arginine starvation. Importantly, even though the AAP3.2 mRNA levels in $\triangle a p 3 D 6$ were similar to WT levels in both the presence and absence of arginine, AAP3 protein levels were not up-regulated in response to arginine deprivation because of the stop codons in the AAP3.2 gene. Two other ADR-responsive genes(Goldman-Pinkovich et al., 2016), LinJ.10.1450 and LinJ.36.2900 (which encode pteridine and MSF family transporters, respectively), responded normally to arginine deprivation in all mutants (Fig. S2), indicating that the CRISPR/Cas9 mutations affected only the response of AAP3.2 to arginine deprivation, not the entire ADR pathway.

The analyses described above indicated that $\triangle a p 3 D 6$ and $\triangle a p 3 D 10$ are the most informative AAP3 mutants, having retained near-WT basal levels of arginine transport (when grown in normal medium), but being unable to up-regulate transporter activity after arginine deprivation. Therefore, we decided to pursue virulence studies with these two mutants to assess whether increased AAP3 expression is essential for infectivity and virulence. We infected THP1 macrophages with late-log phase L. donovani promastigotes of WT, $\triangle a p 3 D 6$, $\triangle a p 3 D 10$ and $\triangle a p 3 G 3-17$ in medium containing a physiologically-relevant concentration of arginine (0.1 mM) (Brown et al.)'(Pawar et al., 2019). As shown in Fig. 3A, the initial (after 4 hours of co-incubation) level of infection (i.e. the percentage of macrophages infected, upper panel) and parasitemia (i.e. the average number of parasites per macrophage, lower panel) with all three mutants were similar to those of WT. However, by 48 hours post-infection, the infectivity and parasitemia for both $\triangle a p 3 D 6$ and $\triangle a p 3 D 10$ were reduced by 2-fold or more compared to WT, while the levels in $\triangle a p 3 \mathrm{G} 3-17$ were comparable to WT (Fig. 3B). Hence, 
our results indicate that the mutants are able to infect macrophages (almost) as well as WT, they fail to proliferate normally thereafter. Indeed, it appears that significant number of macrophages are able to completely clear their internalized parasites, while the parasites in the remaining infected macrophages undergo only one or two rounds of replication (compared to the normal 3-4 rounds of replication and re-infection of new macrophages seen with WT parasites).

As we have previously shown (Pawar et al., 2019), 24 hours after infection of macrophages grown in a medium containing $0.1 \mathrm{mM}$ arginine, amastigotes activate the ADR likely due to arginine depletion in the phagolysosome of infected macrophages. Thus, we used qRT-PCR to quantify mRNA levels in promastigotes immediately before infection of THP1 cells and amastigotes $48 \mathrm{~h}$ after infection. As expected, WT amastigotes showed a significant ( 3-fold) increase in AAP3.2 mRNA levels when compared to promastigotes, but the AAP3.2 mRNA levels in amastigotes from the $\triangle a p 3 D 6$ and $\triangle a p 3 D 10$ mutants were substantially lower than WT. In addition, it should be remembered that the AAP3.2 mRNA in the former is non-functional due to in-frame stop codons. In contrast, AAP3.2 mRNA levels in the $\triangle a p 3 \mathrm{G} 3-17$ mutants (even promastigotes) were higher than WT amastigotes. To assess whether the lower AAP3 expression levels (and consequently less arginine import) was responsible for the reduction in infectivity and parasitemia of the $\triangle a p 3 D 6$ and $\triangle a p 3 D 10$ mutants, we infected THP1 macrophages grown in medium containing $1.5 \mathrm{mM}$ arginine, a concentration that prevented ADR in intracellular WT amastigotes (Pawar et al., 2019). Under these conditions, both $\triangle a p 3 D 6$ and $\triangle a p 3 D 10$ developed normally into amastigotes, with similar infectivity and parasitemia at 48 hours to that of WT (Fig. 3C). The results indicate that the inability to develop inside macrophages is due to arginine deprivation that developed in macrophage phagolysosomes during infection and that the ability to respond to 
this deprivation by up-regulating AAP3.2 protein levels is essential for successful intracellular Leishmania development.

Figure 3

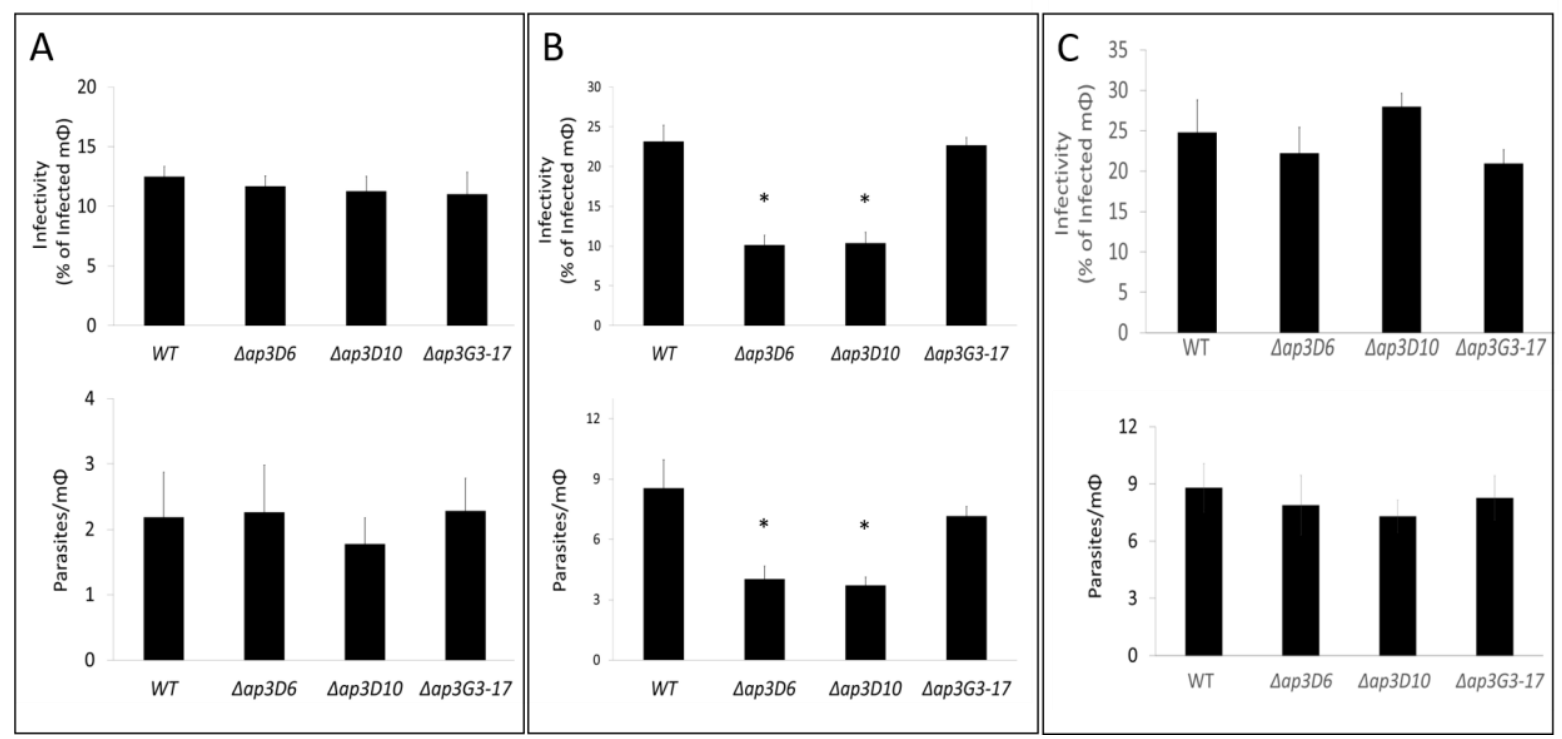

To further assess the role of arginine transport in vivo, we infected BALB/c mice with $\triangle a p 3 D 6, \triangle a p 3 D 10, \triangle a p 3 G 3-17$ and WT (8 mice for each group). On day 21 post-infection, the mice were sacrificed and parasitemia determined by qPCR on the DNA extracted from the liver of each mouse. As shown in Fig. 4, the parasitemia of the $\triangle a p 3 D 6$ and $\triangle a p 3 D 10$ mutants were only 20 and 24\%, respectively, that of WT. One-way ANOVA and Tukey posthoc HSD testing showed a significant difference $(\mathrm{P}<0.001)$ from WT for both the $\triangle a p 3 D 6$ and $\triangle a p 3 D 10$ mutants, while $\triangle a p 3 G 3-17$ was not significantly different from WT ( $\mathrm{p}=0.387)$. These results show that inability of the mutants to express higher levels of AAP3 in order to compensate for the reduced level of arginine in the phagolysomes of infected macrophages severely compromised their virulence. Therefore, it appears that the ADR is a crucial mechanism for enabling intracellular Leishmania parasites to overcome the arginine bottleneck and win the "Hunger Games" in the mammalian host. 
Figure 4

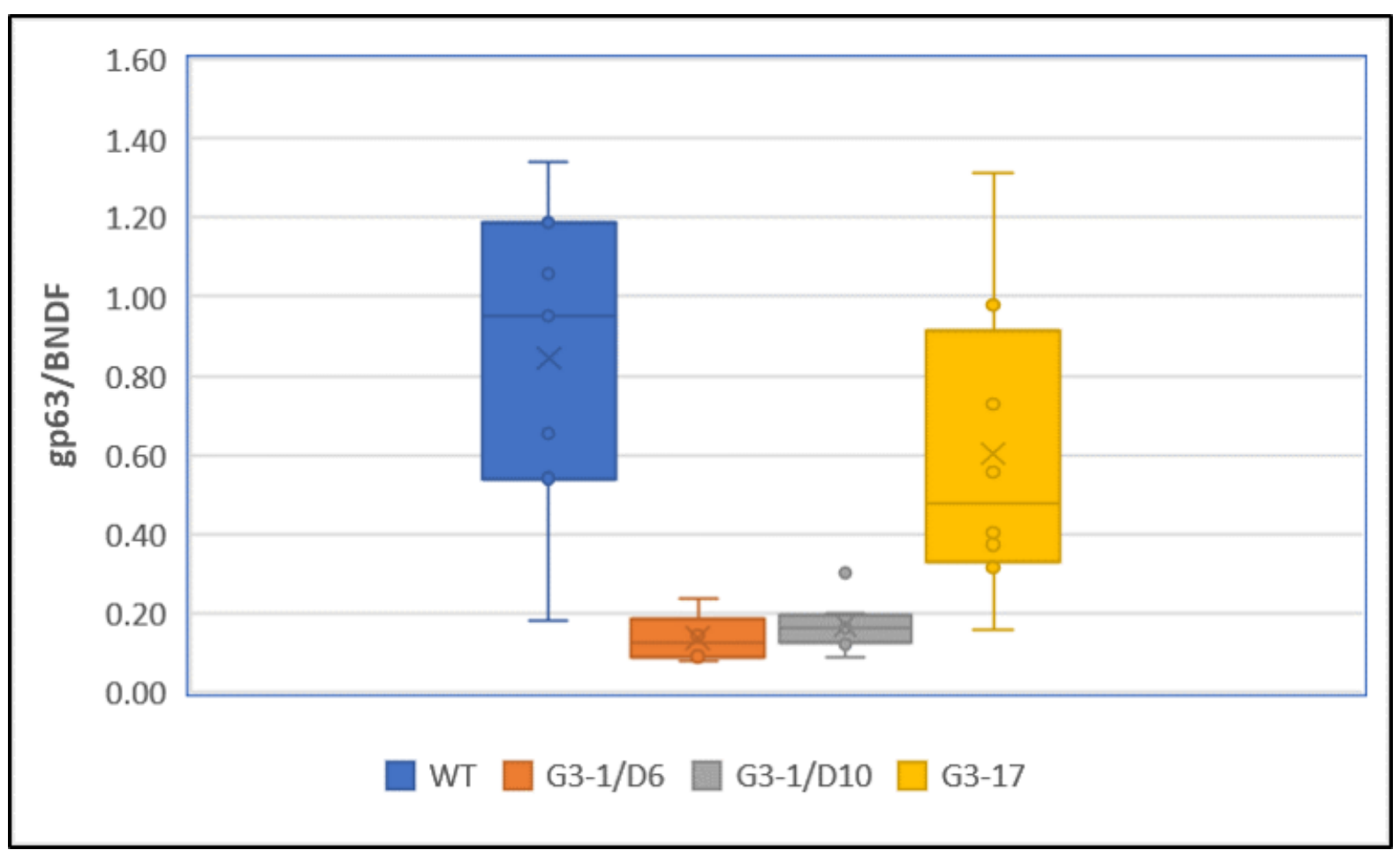

In summary, this study demonstrates for the first time that the ability to monitor metabolic deprivation and subsequently induce a specific response at the level of gene expression is essential for pathogenesis of a protozoan parasite. Furthermore, we have shown that in order for pathogenic microorganisms to respond to host-inflicted environmental changes and survive, they must employ external sensing and response mechanisms that serve as their monitoring device. 


\section{Materials and Methods}

\section{Leishmania strains and culture}

L. donovani MHOM/SD/00/1S (Saar et al., 1998) promastigotes were grown in Medium M199, Earle’s salts (Biological Industries Ltd) supplemented with 10\% heat-inactivated Fetal Bovine Serum (GIBCO Ltd) and 1\% Penicillin-Streptomycin solution (Biological Industries Ltd). Arginine deprivation was carried out as described by Pawar et al. (Pawar et al., 2019). Briefly, mid-log phase promastigotes $\left(1 \times 10^{7}\right.$ cells $\left./ \mathrm{ml}\right)$ were washed with Earle's balanced salt solution twice and re-suspended in arginine-deficient Medium M-199 (Biological Industries Ltd.) at $26^{\circ} \mathrm{C}$ for the specified period before being transferring to ice. Argininedeprived cells were washed twice with ice-cold Earle's balanced salt solution before being used for transport assays, Northern and Western blot analysis.

\section{CRISPR/Cas 9 guide and donor transfections}

gRNA sequences were designed using the Eukaryotic Pathogen CRISPR guide RNA/DNA Design Tool (EuPaGDT, http://grna.ctegd.uga.edu/) and cloned into Leishmaniaadapted vector $\mathrm{pLdCN}$ using the single-step digestion-ligation cloning protocol previously described (Zhang and Matlashewski, 2015), and the constructs transfected into mid-log phase promastigotes. Following gRNA-pLdCN transfections, cells were grown for 4 weeks with G418 at $50 \mu \mathrm{g} / \mathrm{ml}$ and subsequently screened for their ability to increase LdAAP3 protein abundance after arginine deprivation. Next, the G3-donor sequence was introduced into a gRNA G3-originated clone exhibiting the desired phenotype. The G3-donor is a single strand oligo donor (sense) containing 25-nt sequences flanking the Cas9 cleavage site (shown in bold below) and an 11-nt sequence with two in-frame stop codons (underlined and italicized below). Three transfections with $10 \mu \mathrm{l}(100 \mu \mathrm{m})$ of this oligo were performed on 
promastigotes at three day intervals as previously described (Zhang and Matlashewski, 2015). gRNA sequences.

gRNA G1: GTCTATTCCAGCACAGGCGG

gRNA G2: GCCGTCGATAAACACCCGAG

gRNA G3: GTGCCGACGCCGCCAAGCCG

G3-based donor:

ATGAAAACGGTGCCGACGCCGCCAAGTGAGTAGGTAGCCGGGGCGCAACATCA TCTTCCG

\section{Western Blot Analyses}

Western blot analysis was done as described previously(Darlyuk et al., 2009) using a 1:1000 dilution of rabbit anti AAP3 N-terminus antisera(Goldman-Pinkovich et al., 2016).

\section{$\underline{\text { Transport assays }}$}

Uptake of $25 \mu \mathrm{M} \mathrm{L}-\left[{ }^{3} \mathrm{H}\right]$ arginine $(600 \mathrm{mCi} / \mathrm{mmol})$ into L. donovani promastigotes was determined using the rapid filtration technique described previously(Goldman-Pinkovich et al., 2016). Briefly, transport reaction mixture contained $1 \times 10^{8}$ cells $/ \mathrm{ml}$ suspended in Earle's balanced salt solution at pH 7 supplemented with $5 \mathrm{mM}$ glucose and $1 \%$ dialyzed heatinactivated fetal calf. Cells were pre-warmed at $30^{\circ} \mathrm{C}$ for $10 \mathrm{~min}$ prior to addition of radiolabeled arginine. After $0,0.5,1,1.5$ and 2 minutes, the cell suspensions were filtered through GF/C filters. The amount of radiolabel associated with the cells was linear with time over the 2-min time course of the assay, so the initial rate of transport was calculated from the slope of the line fitted by linear regression (Fig. S1). 


\section{$\underline{R N A}$ isolation and real time quantitative reverse transcription $P C R$}

RNA was isolated using TRI reagent® (Sigma-Aldrich Ltd) or Direct-zol RNA

MiniPrep Kit (Zymo Research), according to manufacturer instructions. Eluted RNA samples were quantified using a NanoDrop One spectrophotometer (Thermo Scientific). Two $\mu \mathrm{g}$ of the extracted RNA were subjected to DNase treatment using RQ1 (Promega). Successful DNase treatment was verified by PCR to make sure no residual DNA could be responsible for amplification. cDNA was synthesized from $2 \mu \mathrm{g}$ of DNase-treated RNA using a qScript cDNA Synthesis Kit (Quanta Biosciences) in $40 \mu 1$ total volume. qRT-PCR was carried out with the reagents of SsoAdvanced Universal SYBR Green Supermix (Bio-Rad, Ltd.) in $10 \mu 1$ reaction volume (5 $\mu 1 \mathrm{SYBR}$ Green, $0.5 \mathrm{nM}$ forward primers, $0.5 \mathrm{nM}$ reverse primers and 2.5 $\mu$ cDNA template) on CFX96 Touch Real-Time PCR system (Bio-Rad). The AAP3 primers matched both AAP3.1 and AAP3.2. Primers specific for a regulatory subunit of PKA (PKAR') were used as a control. All the samples were run in triplicates, including a no-template (negative) control for all primers used. Also, RNA-seq data of Arginine Deprivation Response indicated PKAR' is not affected by ADR thus serving as a good control for testing LdAAP3 behavior under ADR and related conditions.

\begin{tabular}{|l|l|l|r|}
\hline LinJ.31. 0910 & AAP3 & Forward & 5'-GGCTTCATCTTCCCTGCGTA-3' \\
LdBPK_3109010.1 & Amplicon: 137 bp & Reverse & 5'-CGGTCGAAATGGTGCCAAAC-3' \\
\hline LinJ.34.2680 & PKAR' & Forward & 5-AGACCGCGAAGCTCAGTC-3' \\
LDBPK_342680.1 & Amplicon: 239 bp & Reverse & 5'-TTGTCGCAGAGAGTACCG-3' \\
\hline LinJ.10.1450 & Pteridine transporter & Forward & 5'-TGCATGTGCGGCATCTTTGGA-3' \\
LdBPK_101450.1 & Amplicon: 171 bp & Reverse & 5'-GCACAGGATAACCGAGATGC-3' \\
& & & \\
\hline
\end{tabular}




\begin{tabular}{|l|l|l|r|}
\hline LinJ.36.2900 & $\begin{array}{l}\text { Nodulin-like/MFS } \\
\text { transporter }\end{array}$ & Forward & 5'-GTCATCGGGCTGGCCAAG-3' \\
LdBPK_362900.1 & Amplicon: $160 \mathrm{bp}$ & Reverse & 5'-GAGGCAGTGCAATGAGAAGC-3' \\
& & \\
\hline
\end{tabular}

PCR was performed at $95^{\circ} \mathrm{C}$ for 30 seconds, followed by 39 cycles of $95^{\circ} \mathrm{C}$ for 10 seconds, $60^{\circ} \mathrm{C}$ for 30 seconds and Melt Curve analysis was carried out at $65-95^{\circ} \mathrm{C}$ with $0.5^{\circ} \mathrm{C}$ increment for $5 \mathrm{sec} / \mathrm{step}$ and data analysis was carried out as described previously(Pfaffl, 2001). Briefly, $\mathrm{C}_{\mathrm{P}}$ values were obtained from all samples in triplicate (except negative controls). Primers were calibrated on pooled samples and primer efficiencies $\left(\mathrm{E}_{\text {target }}\right.$ and $\left.\mathrm{E}_{\mathrm{ref}}\right)$ were calculated and incorporated in the equation below where LdAAP3, Pteridine or MFS transporters were the target gene and PKAR' served as the reference gene. Ratios were calculated, with $\mathrm{C}_{\mathrm{P}}$ of 0 hours or 48 hours infected THP-1 macrophages. WT L. donovani $48 \mathrm{~h}$ infected macrophages served as control.

$$
\text { ratio }=\frac{\left(E_{\text {target }}\right)^{\Delta \mathrm{CP}_{\text {target }}(\text { control }- \text { sample })}}{\left(E_{\text {ref }}\right)^{\Delta \mathrm{CP}_{\text {ref }}(\text { control }- \text { sample })}}
$$

\section{Whole Genome Sequencing}

Genomic DNA was prepared from Leishmania promastigotes using proteinase K digestion followed by phenol/chloroform extraction and ethanol precipitation and fragmentation to 200bp using a Covaris S2 sonicator according to the manufacturer's protocol. NGS libraries were prepared using an NEB NextUltra II kit, quantified on Agilent Bioanalyzer and Qubit instruments and sequenced on an Illumina Hiseq 4000 to generate 4696 million paired-end 75-base pair reads, depending on the sample. Reads were aligned to the L. donovani $1 \mathrm{~S}$ genome sequence assembled from a combination of PacBio and Illumina 
reads (manuscript in preparation) using Bowtie2 within Geneious and gene copy number estimated by normalizing the read counts per gene to account for library size and assuming 4 copies of $\operatorname{chr} 31$.

\section{Infectivity assays}

THP-1 cells were seeded on glass coverslips $\left(1 \times 10^{6}\right.$ cells/well $)$ in a 6 -well plate and treated with $50 \mathrm{ng} / \mathrm{ml}$ of PMA (Sigma-Aldrich, USA) for $48 \mathrm{~h}$. They were infected as described previously (Pawar et al., 2019) and the intracellular parasite load was visualized by Giemsa staining. 


\section{$\underline{\text { In vivo } B A L B / \text { c mice infections and analysis }}$}

L. donovani infections were done by tail intravenous (IV) injection of $10^{8}$ stationary phase promastigotes per mouse. On day 21 of the infection the mice were sacrificed and mouse liver DNA isolated using the protein K method (Hofstetter et al., 1997). Quantitative PCR was performed on mouse liver DNA using mouse BDNF as reference genes and parasite gp63 as target gene with published primer sequences (Tiwananthagorn et al., 2012)'(Tupperwar et al., 2008).

Q-PCR was carried out with the reagents of SYBR Green (ThermoFisher Scientific, Catalog No A25776) in $10 \mu \mathrm{l}$ reaction volume (5 $\mu \mathrm{l}$ SYBR Green, primer final concentration of $300 \mathrm{nM}$ forward primer, $300 \mathrm{nM}$ reverse primer and 125ng DNA template) on CFX96 Real-Time PCR system (Bio-Rad). All samples were run in triplicate separately for the 2 primer sets and the PCR cycle include $30 \mathrm{~s}$ incubation step at $95^{\circ} \mathrm{C}$, then 40 cycles of $5 \mathrm{~s}$ at $95^{\circ} \mathrm{C}$ and $30 \mathrm{~s}$ at $60^{\circ} \mathrm{C}$. The output of normalized expression was determined by the BIORAD software of the instrument. 


\section{Acknowledgments}

We thank Prof. Ido Izhaki of Haifa University for his help with the statistical analyses and MS Michal Almoznino for experimental analyses.

Funding for this work was provided by the University Grant Commission (UGC) - Israel

Science Foundation (Indo-Israel) research grant number 2316/15 to DZ, AG and RM and USA-Israel Binational Foundation grant number 2017030 to DZ and PJM. 


\section{Figure Legends}

Figure 1: Targeting CRISPR guides to the 5' end of $A A P 3$ ORF impaired their response to arginine deprivation

Leishmania-adapted CRISPR/Cas9 targeted to the 5' end of AAP3 ORF at positions 19, 85 and $173 \mathrm{bp}$ into the CDS with gRNAs designated G1, G2 and G3, respectively (A, top panel). gRNA-transfected promastigotes were grown in culture, supplemented with neomycine $(50 \mu \mathrm{g} / \mathrm{ml})$, for six weeks. Subsequently, subjected protein extracted from these cells were subjected to western analysis using anti LdAAP3 antiserum (and HSP83 antisera for loading control, B, top left panel). G3 cells (highlighted in square) then seeded on agar plates and three types of colonies raised, $\triangle a p 3 G 3-1$, $\triangle a p 3 \mathrm{G} 3-2$ and $\triangle a p 3 \mathrm{G} 3-17$ (B, top right panel). $\triangle a p 3 G 3-1$ (highlighted in square) was then further transfected with a donor, i.e. the gRNA G3 based sequence containing an 11-bp insertion with stop codons (A, bottom panel). These transfectants gave rise to several colonies of $\Delta(a a 3-a d r)$ mutants that we named $\triangle a p 3 D 4, \triangle a p 3 D 6, \triangle a p 3 D 7$ and $\triangle a p 3 D 10$ (B, bottom panel). Fold change of initial rate of arginine transport (2 minutes, Fig. S1) before and after two hours after arginine starvation $(\mathbf{C}$, blue and red, respectively). (D) Northern analysis of AAP3 mRNA expression before and after two hours of arginine starvation.

\section{Figure 2: Whole genome sequencing of the AAP3 region in the CRISPR/Cas9 treated L. donovani promastigotes}

Whole genome sequencing carried out for each of the WT and $\Delta$ (aap3-adr) mutants. Illumina libraries were constructed and sequenced on a HiSeq 4000 using paired-end 75-bp reads with TruSeq standard primers. (A) Reads were aligned against the Ldo1S reference genome and revealed that mutants $\triangle a p 3 G 3-1, \triangle a p 3 D 4$ and $\triangle a p 3 D 10$ harbor a large deletion essentially fusing AAP3.1 CDS with that of AAP3.2 and eliminating AAP3.2 3'UTR and all intergenic ncRNA sequences. Quantification of reads indicates that gene fusion event has occurred on two copies of chromosome 31 in $\triangle a p 3 G 3-1$, 
three copies in $\triangle a p 3 D 4$ and on four copies in $\triangle a p 3 D 10 . \triangle a p 3 G 3-17$ harbors 16 copies of the region. $\triangle a p 3 D 6$ alignment to a version of $\operatorname{LdoS} \_31$ revealed an 11-bp insertion (with a stop codon in each frame) in the AAP3.2 gene. The read counts for each gene represent only those that can be unambiguously assigned to the WT or mutant version of the gene. These results are consistent with the hypothesis that 3 copies of AAP3.2 and 3 copies of the 4 AAP3.1 genes contain stop codons in $\triangle a p 3 D 6$ (A). Reads analysis indicated that neighboring genes such as LinJ.31.0890 and LinJ. 0920 were not affected by the AAP3 mutation $(\mathbf{B})$.

\section{Figure 3: Mutants lacking AAP3-ADR lost infectivity to THP-1 macrophages}

Human THP-1 differentiated macrophages grown in RPMI1640 medium containing $0.1 \mathrm{mM}$ arginine, subjected to infection by mid-log L. donovani promastigotes of WT, $\triangle a p 3 D 6, \triangle a p 3 D 10$ and $\triangle a p 3 G 3-$ 17 at an MOI of 10 for four hours, as described in Methods. At times zero and 48 hours after infection macrophages were fixed, giemsa stained and subsequently subjected to counting. Virulence capacity was determined by calculating infectivity (A and B top panels) and parasitemia (A and B bottom panels). Macrophage infections carried out in the presence of $0.1 \mathrm{mM}$ arginine (A and $\mathrm{B}$ ) or $1.5 \mathrm{mM}$ (C). For panel B, one-way ANOVA indicated that $\triangle a p 3 D 10$ and $\triangle a p 3 D 6$ mutants were $57 \%$ less infective than WT $(\mathrm{p}<0.05, \mathrm{n}=16)$ and parasitemia 50\% less than WT $(\mathrm{p}<0.05, \mathrm{n}=4)$. When infection was performed at $1.5 \mathrm{mM}$ external arginine concentration One-way ANOVA was insignificant for mutant impairment in either infectivity $(\mathbf{C}$, top panel) or parasitic burden $(\mathbf{C}$, bottom panel $)$ parameters $(\mathrm{p}<0.1)$.

\section{Figure 4. AAP3.2 mutants are unable to develop in mice}

Late stationary phase L. donovani promastigotes of WT, Aap3D6, $\triangle a p 3 D 10$ and $\triangle a p 3 G 3-17$ injected intravenously (IV) into eight-week-old female $\mathrm{BALB} / \mathrm{c}$ mice $\left(1 \mathrm{X} 10^{8}\right.$ cells per injection, eight mice per mutant). On day21 post infection, mice were sacrificed and liver DNA extracted using the proteinase K method(Hofstetter et al., 1997). Analyses were carried out as described in Methods. 


\section{Supplementary Figures}

A

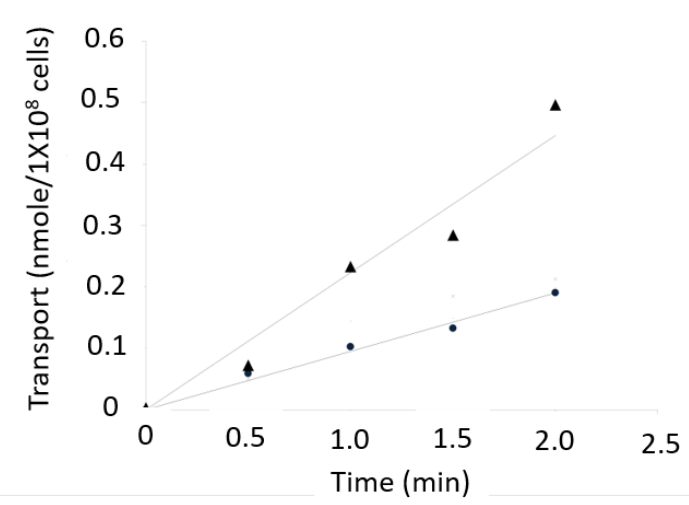

B

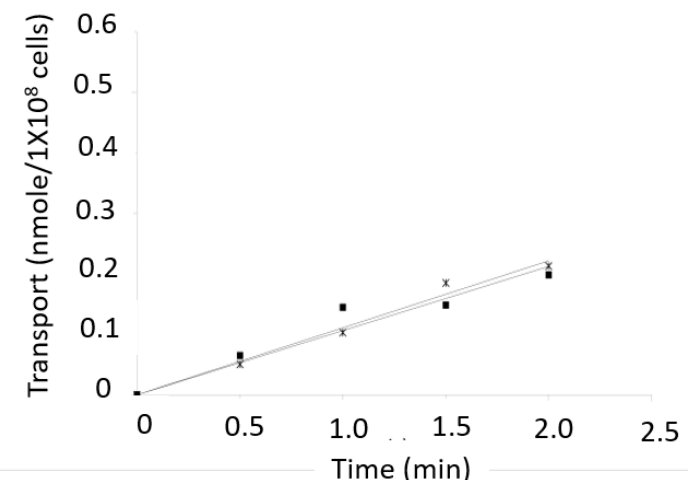

Figure S1: Initial rate of arginine transport in wild type and mutant L. donovani promastigotes

Two minutes arginine transport was determined using the rapid filtration technique (Methods).

Transport was assayed in wild type before $(\bullet)$ and after $(\boldsymbol{\Delta})$ two hours arginine starvation and in $\triangle a p 3 D 6$ before $(\bullet)$ and two hours after arginine starvation (*). 
A

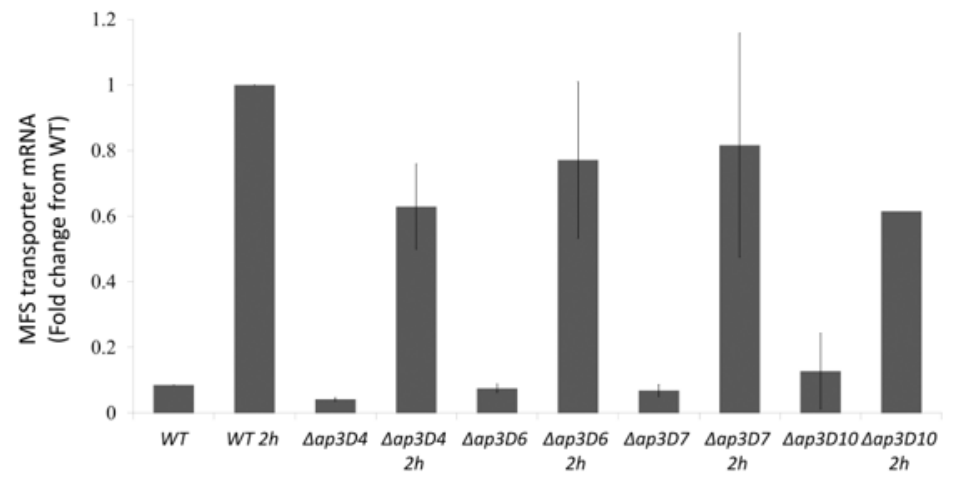

B

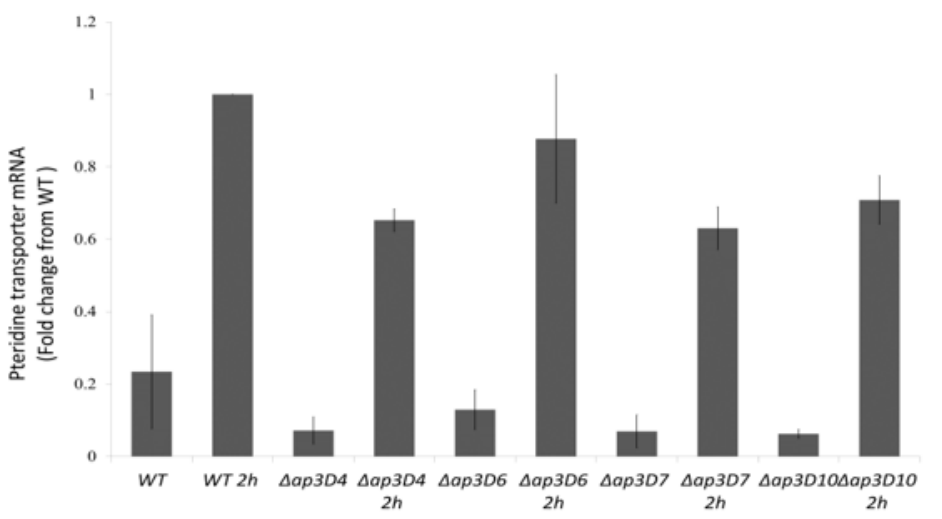

Figure S2: Other members of ADR retained sensitivity to arginine deprivation

Real Time PCR analysis was carried out to determine mRNA levels of pteridine (upper panel) and MSF (lower panel) transporters before and after two hours after arginine starvation. The analyses were carried out in $\triangle a p 3 D 4, \triangle a p 3 D 6, \triangle a p 3 D 7$ and $\triangle a p 3 D 10$. Results are relative to 2 hour arginine deprived WT cells. 


\section{References}

Brown, C.M., Becker, J.O., Wise, P.M., and Hoofnagle, A.N. Simultaneous determination of six L-arginine metabolites in human and mouse plasma using hydrophilic-interaction chromatography and electrospray-tandem mass spectrometry.

Darlyuk, I., Goldman, A., Roberts, S.C., Ullman, B., Rentsch, D., and Zilberstein, D. (2009). Arginine homeostasis and transport in the human pathogen Leishmania donovani. J Biol Chem 284, 19800-19807.

Downing, T., Imamura, H., Decuypere, S., Clark, T.G., Coombs, G.H., Cotton, J.A., Hilley, J.D., de Doncker, S., Maes, I., Mottram, J.C., et al. (2011). Whole genome sequencing of multiple Leishmania donovani clinical isolates provides insights into population structure and mechanisms of drug resistance. Genome Res 21, 2143-2156.

Goldman-Pinkovich, A., Balno, C., Strasser, R., Zeituni-Molad, M., Bendelak, K., Rentsch, D., Ephros, M., Wiese, M., Jardim, A., Myler, P.J., et al. (2016). An Arginine Deprivation Response Pathway Is Induced in Leishmania during Macrophage Invasion. PLoS Pathog 12, e1005494.

Hofstetter, J.R., Zhang, A., Mayeda, A.R., Guscar, T., Nurnberger, J.I., and Lahiri, D.K. (1997). Genomic DNA from mice: a comparison of recovery methods and tissue sources. Biochem. Mol. Med. 62, 197-202.

Laffitte, M.N., Leprohon, P., Papadopoulou, B., and Ouellette, M. (2016). Plasticity of the Leishmania genome leading to gene copy number variations and drug resistance. F1000Res 5,2350 .

Pawar, H., Puri, M., Fischer Weinberger, R., Madhubala, R., and Zilberstein, D. (2019). The arginine sensing and transport binding sites are distinct in the human pathogen Leishmania. PLoS Negl. Trop. Dis. 13, e0007304.

Pfaffl, M.W. (2001). A new mathematical model for relative quantification in real-time RT- 
PCR. Nucleic Acids Res. 29, 45e-45.

Rath, M., MÃ̄1/4ler, I., Kropf, P., Closs, E.I., and Munder, M. (2014). Metabolism via Arginase or Nitric Oxide Synthase: Two Competing Arginine Pathways in Macrophages. Front. Immunol. 5, 532.

Saar, Y., Ransford, A., Waldman, E., Mazareb, S., Amin-Spector, S., Plumblee, J., Turco, S.J., and Zilberstein, D. (1998). Characterization of developmentally-regulated activities in axenic amastigotes of Leishmania donovani. Mol Biochem Parasitol 95, 9-20.

Saxton, R.A., and Sabatini, D.M. (2017). mTOR Signaling in Growth, Metabolism, and Disease. Cell 168, 960-976.

Shahi, M., Mohajery, M., Shamsian, S.A., Nahrevanian, H., and Yazdanpanah, S.M. (2013). Comparison of Th1 and Th2 responses in non-healing and healing patients with cutaneous leishmaniasis. Rep Biochem Mol Biol 1, 43-48.

Tiwananthagorn, S., Iwabuchi, K., Ato, M., Sakurai, T., Kato, H., and Katakura, K. (2012). Involvement of $\mathrm{CD}^{+} \mathrm{Foxp}^{+}$regulatory $\mathrm{T}$ cells in persistence of Leishmania donovani in the liver of alymphoplastic aly/aly mice. PLoS Negl. Trop. Dis. 6, e1798.

Tupperwar, N., Vineeth, V., Rath, S., and Vaidya, T. (2008). Development of a real-time polymerase chain reaction assay for the quantification of Leishmania species and the monitoring of systemic distribution of the pathogen. Diagn. Microbiol. Infect. Dis. 61, 23-30. Weinstein, S.L., Finn, A.J., Dave, S.H., Meng, F., Lowell, C.A., Sanghera, J.S., and DeFranco, A.L. (2000). Phosphatidylinositol 3-kinase and mTOR mediate lipopolysaccharide-stimulated nitric oxide production in macrophages via interferon-beta. $\mathbf{J}$ Leukoc Biol 67, 405-414.

Zhang, W.W., and Matlashewski, G. (2015). CRISPR-Cas9-Mediated Genome Editing in Leishmania donovani. MBio 6, e00861. 